OPEN ACCESS

Edited by:

Dongsheng Zhou,

Beijing Institute of Microbiology and Epidemiology, China

Reviewed by:

Muhammad Nawaz, University of Veterinary and Animal

Sciences, Pakistan

Yanwen Xiong,

National Institute for Communicable

Disease Control and Prevention,

China

Jiru Xu,

Xi'an Jiaotong University, China

Marisa Haenni,

National Agency for Sanitary Safety

of Food, Environment and Labor

(ANSES), France

${ }^{*}$ Correspondence:

$\mathrm{LiBa}$

baili@cfsa.net.cn

Zengqi Yang

yzq8162@163.com

tThese authors have contributed equally to this work

Specialty section: This article was submitted to Antimicrobials, Resistance and Chemotherapy,

a section of the journal Frontiers in Microbiology

Received: 11 June 2019 Accepted: 14 August 2019 Published: 29 August 2019

Citation: Zhang P, Wang J, Wang X, Bai $X$ Ma J, Dang R, Xiong Y, Fanning S, Bai $L$ and Yang Z (2019)

Characterization of Five Escherichia coli Isolates Co-expressing ESBL and MCR-1 Resistance Mechanisms

From Different Origins in China. Front. Microbiol. 10:1994. doi: 10.3389/fmicb.2019.01994

\section{Characterization of Five Escherichia coli Isolates Co-expressing ESBL and MCR-1 Resistance Mechanisms From Different Origins in China}

\author{
Pei Zhang ${ }^{1,2,3+}$, Juan Wang ${ }^{1 \dagger}$, Xinglong Wang ${ }^{1}$, Xue Bai ${ }^{1}$, Jiangang Ma ${ }^{1}$, Ruyi Dang ${ }^{1}$, \\ Yifei Xiong ${ }^{4}$, Séamus Fanning ${ }^{3,5}$, Li Bai $^{3 *}$ and Zengqi Yang ${ }^{1 *}$ \\ ${ }^{1}$ College of Veterinary Medicine, Northwest A\&F University, Xianyang, China, ${ }^{2}$ Guangdong Provincial Key Laboratory of \\ Food, Nutrition and Health, School of Public Health, Sun Yat-sen University, Guangzhou, China, ${ }^{3}$ NHC Key Laboratory of \\ Food Safety Risk Assessment, China National Center for Food Safety Risk Assessment, Beijing, China, ${ }^{4}$ College of Food \\ Science and Engineering, Northwest A\&F University, Xianyang, China, ${ }^{5}$ UCD-Centre for Food Safety, School of Public \\ Health, Physiotherapy and Sports Science, University College Dublin, Dublin, Ireland
}

Present study characterized five Escherichia coli co-expressing ESBL and MCR-1 recovered from food, food-producing animals, and companion animals in China. Antimicrobial susceptibility tests, conjugation experiments, and plasmid typing were performed. Whole genome sequencing (WGS) was undertaken for all five isolates using either PacBio RS II or Illumina HiSeq 2500 platforms. The cefotaxime and colistin resistance encoded by blacTX-M and mcr-1 genes, respectively, was transferable by conjugation either together or separately for all five strains. Interestingly, the ESBL and mcr-1 genes could be co-selected by cefotaxime, while the colistin only selected the mcr-1-carrying plasmids during the conjugation experiments. Five E. coli sequence types (ST88, ST93, ST602, ST162, and ST457) were detected. Although diverse plasmid profiles were identified, Incl2, IncFIB, and IncFIl plasmid types were predominant. These five clonally unrelated isolates harbored the mcr-1 gene located on similar plasmid backbones, which showed high nucleotide similarity to plasmid pHNSHP45. The mcr-1 gene can be co-transmitted with blaCTX-M genes through Incl2 plasmids with or without ISAp/1 in our study. Characterization of these co-existence ESBL and mcr-1 isolates extends our understanding on the dissemination of these resistance markers among bacteria of diverse origins.

Keywords: ESBL, co-occurrence, conjugative plasmids, mcr-1 gene, Escherichia coli

\section{INTRODUCTION}

Multidrug resistant (MDR) Enterobacteriaceae isolates have been widely reported in non-clinical environments, from pets and farm animals as well as food, which increases the risk for humans regarding the foodborne or zoonotic transmission routes. The emergence of transferable polymyxin resistance mediated by $m c r-1$ represents a threat to the revival of polymyxin E, which has 
emerged as our last-resort drug for treating carbapenemresistant Enterobacteriaceae infections (Michalopoulos et al., 2005; Liu et al., 2016; Paterson and Harris, 2016). Since the first plasmid-mediated $m c r-1$ gene was reported in members of the Enterobacteriaceae family isolated from humans and food-producing animals in China in late 2015, $\mathrm{mcr}-1$ has been recognized in over 50 countries/regions covering five continents (Liu et al., 2016; Sun et al., 2018). Several retrospective studies have reported mcr-1 gene in Escherichia coli, Escherichia albertii, Klebsiella pneumonia, and Salmonella species from different geographical locations, with the earliest evidence pointing to its existence dating back to the 1980s (Brennan et al., 2016; Figueiredo et al., 2016; Schwarz and Johnson, 2016; Shen et al., 2016).

Mobile genetic elements (MGE), especially plasmids, are considered to be a key driver of horizontal gene transfer (HGT) of the ten $m c r-1$ variants reported to date (Shen et al., 2018). So far, various plasmid replicon types, such as IncI2, IncHI1, IncHI2, IncP, IncFIB and IncX4 have been reported to be associated with HGT of the $m c r$ genes (Doumith et al., 2016; Poirel et al., 2016; Zurfluh et al., 2016). It is unusual, but not without precedent, that $m c r-1$ has already been found to coexist with both ESBL and $b l a_{N D M-1}$ (or its variants $b l a_{N D M-5}$ and $\left.b l a_{N D M-9}\right)$ on these promiscuous plasmids (Du et al., 2016; Haenni et al., 2016; Li Y. et al., 2018). Moreover, the insertion sequence ISApl1 has also been shown to be a key element mediating translocation of $\mathrm{mcr}-1$ into plasmids of diverse replicon types through formation of a circular intermediate (Li et al., 2017). Notably, the ISApl1-mcr1 -cassette was also found to be located on the chromosome, which further supports the hypothesis that ISApl1 might be involved in $m c r-1$ acquisition. Thus investigation of the MGE, especially plasmids and insertion sequences, is a key component required for a better understanding of the dissemination of $\mathrm{mcr}-1$ gene and others.

Our study was initiated by the isolation of five cefotaxime and colistin co-resistant E. coli cultured originally from 541 isolates taken from different regions in China from 2012 to 2016. In this paper, we reported the characterization of five $E$. coli isolates, with the aims of this study being (i) to determine the co-occurrence of ESBL and MCR-1 markers, (ii) to identify the co-transference mechanisms by dual conjugation assays, (iii) to characterize the E. coli by WGS, and (iv) to identify the plasmid backbones and genetic environments containing the $m c r-1$ genes.

\section{MATERIALS AND METHODS}

\section{Bacterial Collection}

In this study, five $m c r-1$ positive and cefotaxime resistant isolates were detected among $265 \mathrm{E}$. coli cultured from retail food $(n=2), 69 E$. coli isolates from sick companion dogs $(n=1)$, $93 \mathrm{E}$. coli isolates from pigs $(n=1)$, and $114 \mathrm{E}$. coli isolates from poultry $(n=1)$. All isolates were collected in geographically distinct regions located in Beijing, Tianjin, Hangzhou, Guiyang, Chongqing cities during 2012-2016 in China. The species of all isolates were determined by $16 \mathrm{~S}$ rDNA sequencing. Primers targeting the $m c r-1$ gene were described previously used to screen the studied isolates (Liu et al., 2016).

\section{Antimicrobial Susceptibility Testing and Detection of Resistance Genes}

The minimum inhibitory concentration (MIC) of colistin (Sigma, St Louis, MO, United States) was determined by broth dilution method according to the European Committee on Antimicrobial Susceptibility Testing (EUCAST) ${ }^{1}$. In addition, all isolates were tested for their susceptibility to a panel of antimicrobial compounds by disk diffusion, following recommendations of the Clinical and Laboratory Standards Institute (Clinical and Laboratory Standards Institute [CLSI], 2016). Reference strain E. coli ATCC ${ }^{\mathrm{TM}} 25922$ served as a quality control. Thereafter, the $\beta$-lactamase-encoding genes belonging to the $b l a_{T E M}, b l a_{C T X}-M$, bla $a_{G E S}$, bla $a_{P E R}$, and bla $a_{V E B}$ families were screened by PCR (Dallenne et al., 2010).

\section{Conjugation Based Mating Experiments and Verification}

Five ESBL- and mcr-1-positive isolates were analyzed individually, for their ability to transfer colistin/cefotaxime resistance to a rifampicin-resistant, plasmid-free E. coli recipient (26R 793) (Wang J. et al., 2016a). Conjugation experiments were carried out in triplicate using a broth mating protocol as described previously (Wang J. et al., 2013). Transconjugants were selected on plates containing $2 \mu \mathrm{g} / \mathrm{mL}$ colistin with $100 \mu \mathrm{g} / \mathrm{mL}$ rifampicin or $4 \mu \mathrm{g} / \mathrm{mL}$ cefotaxime with $100 \mu \mathrm{g} / \mathrm{mL}$ rifampicin. Transfer frequencies were calculated per donor cell. Antimicrobial susceptibility tests were performed to confirm the plasmid transfer as described above, followed by S1-nuclease pulsed-field gel electrophoresis (PFGE) and PCR-based replicon typing (PBRT) to test which plasmids and resistance markers were transferred.

\section{Plasmid Profiling and PCR-Based Replicon Typing}

S1-nuclease-digested (Promega, Madison, WI, United States) linearized plasmid DNAs from the five ESBL and MCR-1 positive isolates and their transconjugants were further examined by PFGE for the detection and size determination of large plasmids as described previously (Barton et al., 1995). The approximate molecular mass of plasmids was determined by comparing with E. coli 39R 861 (Macrina et al., 1978). The isolates were also examined for the presence of 18 replicon types by PBRT (Carattoli et al., 2005).

\section{Whole Genome Sequencing and Phylogenetic Analysis}

To assess the genomic background of the five isolates, whole genome sequencing (WGS) was performed using the PacBio RS II system (for CQ9 isolate) or the Illumina HiSeq 2500 platform (Illumina, San Diego, CA, United States) (for F81, F89, Dog1, and CY69 isolates). Reads from Illumina platform were assembled

\footnotetext{
${ }^{1}$ http://www.eucast.org; accessed March 22, 2016.
} 
de novo using SOAPdenovo ${ }^{2}$. Annotation of the genomes was performed using RAST ${ }^{3}$, BLASTn and BLAST $\mathrm{p}^{4}$ programs. The ORF Finder program ${ }^{5}$ was also used to identify features. Standard methods were used to annotate the serotype, multidrug resistance genes, and plasmid incompatibility types based on the WGS data by CGE platforms ${ }^{6}$. Sequence comparison and map generation were performed using BLAST and Easyfig version 2.1.

\section{Nucleotide Accession Numbers}

Sequences were deposited in GenBank under accession numbers: CQ9 genome (CP031546), pCQ9-1 (CP031547), pCQ9-2 (CP031548), pCQ9-3 (CP031549) and pCQ9-4 (CP031550), F81 (QXGP01000001 to QXGP01000155), F89 (QXGO01000001 to QXGO01000093), Dog1 (QUON01000001 to QUON01000107), and CY69 (QUOM01000001 to QUOM01000089).

\section{RESULTS AND DISCUSSION}

\section{Bacterial Isolation and Molecular Characterization}

The five $(\sim 1 \%)$ cefotaxime- and colistin-resistant isolates were identified from totally $541 \mathrm{E}$. coli strains from animal and food sources of different geographical regions in China from 2012 to 2016 (Table 1). The prevalence is much lower than the data reported earlier ( $\mathrm{Li}$ et al., 2017; Shen et al., 2018;

${ }^{2}$ https://www.animalgenome.org/bioinfo/resources/manuals/SOAP.html

${ }^{3}$ http://rast.nmpdr.org

${ }^{4}$ http://blast.ncbi.nlm.nih.gov/Blast.cgi

${ }^{5}$ http://www.ncbi.nlm.nih.gov/orffinder

${ }^{6}$ https://cge.cbs.dtu.dk/services/PlasmidFinder/
Wu C. et al., 2018), and one possible reason for this could be the diverse sources and different geographical origins of the samples. All five isolates were sub-typed by MLST and the corresponding serotypes identified in silico following analysis of the WGS data. These E. coli isolates were assigned to five different sequence types/serotypes: ST88 (O116:H11), ST93 (O5:H10), ST602 (O18:H11), ST162 (ST469 complex, Ont:H19), ST457 (O3:H25), and suggesting genetic diversity among these isolates. The diversity of sequence-type clades of cefotaxime and colistin co-expressing strains further confirms the wide distribution of ESBLs- and mcr-carrying $E$. coli and that corresponding resistance is mediated mainly by plasmids rather than adapted dominant bacterial clones (Shen et al., 2018).

S1-nuclease plasmid analysis revealed that all five isolates contained high molecular weight plasmids (ranging from approximately 50 - to $\sim 250-\mathrm{kb}$ ); the CQ9 isolate possessed four plasmids, F81 possessed three plasmids, and each of the remaining three isolates each possessed two plasmids (for F89, Dog1, and CY69). Although heterogeneity among the plasmid profiles was a common feature noted, all isolates carried one plasmid of approximately 60-kb in size (Figure 1). Notably, the $60-\mathrm{kb}$ plasmids from all five strains were transferred by conjugation under laboratory conditions after selection by either colistin or cefotaxime.

PBRT and Plasmid finder showed that all isolates possessed at least three plasmid replicons: including the IncI2, IncFIB and IncFII replicon types, which were considered to be the epidemic resistance plasmid replicon types bearing the greatest variety of resistance genes in Enterobacteriaceae (Carattoli, 2011; Rozwandowicz et al., 2018). One isolate, F81 from retail food also carried an IncR plasmid, which is increasingly described as a reservoir of multidrug resistance (Compain et al., 2014;

TABLE 1 | Features of $m c r-1$ positive E. coli strains include the species, serotype, as well as year, original, and MLST are also shown.

\begin{tabular}{|c|c|c|c|c|c|c|c|c|}
\hline Strains & Original & Year & Serotype & MLST & Plasmid finder & MDR genes & $\begin{array}{l}\text { Sequencing } \\
\text { platform }\end{array}$ & Accession number \\
\hline CQ9 & Pig & 2012 & O116:H11 & ST88 & $\begin{array}{l}\text { IncHI2; IncHI2A; } \\
\text { IncFIC(FII); IncFIB; } \\
\text { IncX1; IncFII(29); } \\
\text { IncA/C2; and Incl2 }\end{array}$ & $\begin{array}{l}\text { bla }_{C T \chi}-M-55, \text { mcr-1, } \\
\text { bla } \\
\text { aad } 1 \text {-2 } 1, \text { aph }\left(3^{\prime}\right)-\text { la, erm }(B), \\
\text { mph(A), floR, cmlA1, sul1, sul2, } \\
\text { sul3, tet(A), and dfrA12 }\end{array}$ & $\begin{array}{l}\text { PacBio RS II } \\
\text { system }\end{array}$ & СР031546 \\
\hline F81 & Retail food & 2014 & O5:H10 & ST93 & $\begin{array}{l}\text { IncFIB-69; IncFII-154; } \\
\text { IncFIC(FII)-137; } \\
\text { IncR-51; and Incl2-31 }\end{array}$ & $\begin{array}{l}\text { bla } C T X-M-55, \text { mcr-1, strA } B \text {, } \\
\text { aph(3')-la, aadA2, blaTEM-1B, } \\
\text { qnrS1, oqxA/B, fosA, mph(A), } \\
\text { floR, sul1, sul2, tet(A), and } \\
\text { dfrA12 }\end{array}$ & Illumina MiSeq & $\begin{array}{l}\text { QXGP01000001 to } \\
\text { QXGP01000155 }\end{array}$ \\
\hline F89 & Retail food & 2014 & O18:H11 & ST602 & $\begin{array}{l}\text { IncFIB-47; IncFIC-32; } \\
\text { and Incl2-21 }\end{array}$ & $\begin{array}{l}\text { bla } C T X-M-64, \text { mcr-1, strA } B \text {, } \\
\text { oqxA/B, floR, sul2, tet(A), and } \\
\text { dfrA17 }\end{array}$ & Illumina MiSeq & $\begin{array}{l}\text { QXG001000001 to } \\
\text { QXG001000093 }\end{array}$ \\
\hline Dog1 & Dog & 2016 & Ont:H19 & $\begin{array}{l}\text { ST162 } \\
\text { (ST469 } \\
\text { Cplx) }\end{array}$ & $\begin{array}{l}\text { IncFIB-55; IncFII-30; } \\
\text { Incl2-27 }\end{array}$ & $\begin{array}{l}\text { bla } C T X-M-24, \text { mcr-1, strA } B, \\
\text { aac(3)-Ild, aph(3')-la, } \\
\text { bla } \\
\text { tet(A)-1B, } m p h(A), \text { floR, sul2, }\end{array}$ & Illumina MiSeq & $\begin{array}{l}\text { QUON01000001 to } \\
\text { QUON01000107 }\end{array}$ \\
\hline CY69 & Poultry & 2016 & O3:H25 & ST457 & $\begin{array}{l}\text { IncFIB-47; IncFII-54; } \\
\text { and Incl2-27 }\end{array}$ & $\begin{array}{l}\text { bla } C T X-M-27, \text { mcr-1, aadA2, } \\
\text { aph(3')-la, strAVB, rmtB, } \\
\text { blatEM-1B, fosA, mph(A), } \\
\text { erm(B), floR, sul1, sul2 tet(A), } \\
\text { and dfrA12 }\end{array}$ & Illumina MiSeq & $\begin{array}{l}\text { QUOM01000001 to } \\
\text { QUOM01000089 }\end{array}$ \\
\hline
\end{tabular}




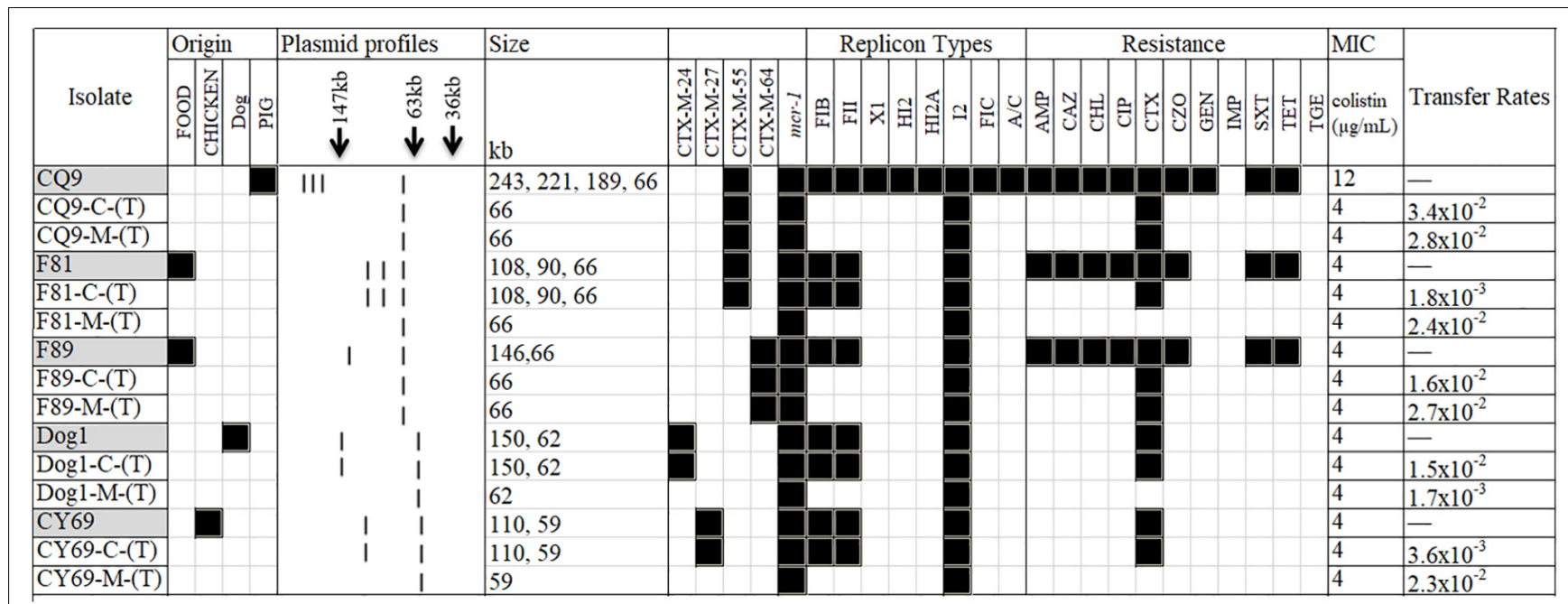

FIGURE 1 | A heat-map showing the comparison of the five Escherichia coli donors and the resultant transconjugants, characterized on the basis of their plasmid profiles according to the S1-PFGE results; ESBL- and mcr-1-markers identified by PCR; antimicrobial resistance profile; and plasmid replicon type(s). The MIC for colistin in each group combination is also shown. ( $\mathrm{T}$ ) in the first column typifies the transconjugant. $\mathrm{C}$ and $\mathrm{M}$ in the first column typifies cefotaxime and colistin, respectively. Black and white squares denote the presence and absence, respectively, of a particular feature. Antimicrobial compounds are abbreviated as follows: AMP, ampicillin; CAZ, ceftazidime; CHL, chloramphenicol; CIP, ciprofloxacin; CTX, cefotaxime; CZO, cefazolin; GEN, gentamicin; IMP, imipenem; SXT,

trimethoprim/sulfamethoxazole; TET, tetracycline; TGE, tigecycline.

Kocsis et al., 2016). Consistent with its plasmid profile, the isolate CQ9 of porcine origin possessed a broader plasmid incompatibility profile, in addition to the ones described above, which also contained the IncHI2, IncX1, and IncA/C2 type plasmids. A summary of these features along with the corresponding antimicrobial resistance profiles for all 5 isolates and their transconjugants is shown in Figure $\mathbf{1}$ and Table 1.

\section{Antibiotic Resistance and Associated Determinants of the ESBL and MCR-1 Co-producing Isolates}

Phenotypic characterization of antibiotic resistance indicated that all the five cefotaxime and colistin co-expressing isolates, also expressed a multidrug-resistant phenotype (resistance to more than two antibiotic classes) and resistance to chloramphenicol, ciprofloxacin and tetracycline in addition to the colistin and $\beta$-lactam antibiotics were recorded (Figure 1). In contrast, all isolates were susceptible to aztreonam, imipenem and cefepime. The MICs of colistin for the donor strains and their transconjugants were between 4 and $8 \mu \mathrm{g} / \mathrm{mL}$ (Figure 1).

Resfinder, using WGS, identified the corresponding molecular mechanisms for antimicrobial resistance phenotypes involved in all five isolates. The genes associated with the colistin resistance were confirmed as mcr-1 gene in all cases. All strains were confirmed as ESBL-producing with different $\operatorname{bla}_{C T X-M}(n=5$, $\left.b l a_{C T X-M-24 / 27 / 55 / 55 / 64}\right)$ and the same $\operatorname{bla}_{T E M-1 B}(n=3)$ genes (Table 1). In addition, CQ9 was the only isolate harbored the widest distribution of the $\operatorname{ampC} C$-like gene $b a_{C M Y-2}$. With the exception of the MDR genes above, the strains were also found to harbor multiple resistance elements, including but not limited to $o q x A B, f l o R, f o s A, d f r A$, erm (B) and $m p h(A)$, which were consistent with the antimicrobial resistance phenotypes in the study (Table 1).

\section{Dual Conjugation Experiments With Antibiotic Selections}

To better address whether the co-transfer of the ESBL and $\mathrm{mcr}$ 1 genes of the isolates could occur, conjugation assays were carried out in triplicate. Five isolates transferred their cefotaxime resistance determinant to a rifampicin-resistant, plasmid-free E. coli $26 \mathrm{R} 793$ recipient with transfer rates ranging from $1.8 \times 10^{-3}$ (for F81) to $3.4 \times 10^{-2}$ (for CQ9) transconjugants per donor cell (Figure 1). The bla $a_{C T X-M}$ genes transferred via conjugation were subsequently confirmed by PCR. Interestingly, the five transconjugants selected by cefotaxime and rifampicin were also resistant to colistin.

Furthermore, to assess the transformation of the colistinresistant phenotype for each donor isolate, an independent conjugation experiment was set up using colistin and rifampicin for selection was also performed. It is notable that all five isolates transferred the colistin resistance marker to the recipient with transfer rates ranging from $1.7 \times 10^{-3}$ (for Dog1) to $2.8 \times 10^{-2}$ (for CQ9) (Figure 1). However, it is very interesting to note that only two transconjugants from CQ9 and F89 were resistant to cefotaxime.

Conjugation studies and plasmid profile analysis of all transconjugants showed that the five donor isolates possessed one plasmid of approximately $60-\mathrm{kb}$. Without exception, these plasmids were all transferred to the recipients. Following PBRT, the transferrable plasmids were identified as belonging to the IncI2 replicon type. Three isolates (F81, Dog1, and CY69) were identified wherein all high molecular weight plasmids were 
transferred via conjugation under the selection by cefotaxime whilst the other two (CQ9 and F89) transferred a single IncI2type plasmid under the selection pressure of either cefotaxime or colistin (Figure 1).

In our study, it is very interesting to note that all of the mcr-1 encoding plasmids can be transferred under the selection pressure of colistin and cefotaxime, but the bla $a_{C T X-M}$ encoding plasmids can only be transferred under the selection-pressure of cefotaxime. Cefotaxime could co-select the ESBL and $\mathrm{mcr}-1$ genes, while the use of colistin only selected the $m c r$-1-carrying plasmids. It is not clear why and how the addition of the two selection antibiotics worked. However, a previous longitudinal study showed that the prevalence of $m c r-1$ was higher in the ESBL-positive E. coli than in the non-ESBL E. coli, and the rapid rising in ESBL prevalence apparently also increased the selective pressure of colistin resistance (Wu C. et al., 2018). Based on these observations, it is tempting to speculate that an antibiotic can affect the overall conjugation dynamics by modulating the conjugation efficiency, serving as a selection force, in itself, that acts on the population dynamics after conjugation, or both (Lopatkin et al., 2016).

\section{Co-occurrence and Co-transference of blactX-M and $\mathbf{m c r}-1$ Genes}

Subsequent plasmid profile analysis showed that all transconjugants contained $1 \sim 3$ detectable large molecularweight plasmids ranging from 58 - to $150-\mathrm{kb}$ in size (Figure 1). Two plasmids were identified from the transconjugants of Dog1C-(T) and CY69-C-(T), but only one plasmid from Dog1-M-(T) and CY69-M-(T) were selected by colistin with rifampicin. Further analysis of the MDR markers indicated the mcr-1 gene and $b l a_{C T X}-M$ in the Dog1 and CY69 isolates were located on two separate plasmids. There were three large plasmids identified from F81. All three were transferrable under the selection of cefotaxime with rifampicin, however, only the smallest plasmid (58-kb in length and $m c r-1$-harboring) was transferred to the recipient under the selection pressure of colistin with rifampicin (Figure 1). In addition to colistin resistance, resistance to several antimicrobial compounds such as ampicillin, chloramphenicol, and cefotaxime were also transferred to the recipient, suggesting that these markers are genetically linked (Figure 1).

One plasmid (66-kb in size) from the strain CQ9 was transferred to the recipients in the two independent conjugation experiments. A similar scenario was noticed in F89, wherein a single plasmid of $63-\mathrm{kb}$ was detected from the transconjugants following selection using cefotaxime with rifampicin and colistin with rifampicin. The cefotaxime resistance determinant was confirmed as bla $a_{C T X}-M-55$ by PCR in CQ9-C-(T) (the corresponding transconjugant selected by cefotaxime with rifampicin) and CQ9-M-(T) (the corresponding transconjugant selected by colistin with rifampicin) and as bla $C T X-M-64$ in F89C-(T) and F89-M-(T), which showed the donor strains of the two had the mcr-1 gene and bla $a_{C T X-M}$ gene co-transferred on the same plasmid (Figure 1). Following PBRT and WGS data analysis, the transferrable and bla $a_{C T X-M}$-harboring plasmids were identified as belonging to the IncI2 replicon type.
It has been shown that the ESBL and $m c r-1$ genes could be co-transferred by one or more types of conjugative plasmid, which could allow their efficient dissemination among bacteria (McGann et al., 2016; Sun et al., 2016, 2017; Wu C. et al., 2018). In our study, two isolates contained the $m c r-1$ gene along with a bla $a_{C T X-M}$ gene $\left(b l a_{C T X-M-55}\right.$ gene from CQ9 and the bla $a_{C T X-M-64}$ gene from F89) and these were co-transferred on the same plasmid, which was confirmed by the conjugation experiments and the sequencing data. In the rest three isolates the ESBL $\left(b l a_{C T X}-M-24 / 27 / 55\right)$ and $m c r-1$ genes were located on two separate plasmids. Such phenomena may contribute to the co-selection of antibiotic resistance carried by multiple types of plasmids and caused by improper combination usage of antibiotics. For the mcr-1-positive transconjugants in the study, the sizes of plasmids observed were approximately 60$\mathrm{kb}$ and all belonged to IncI2 which is consistent with the previous study of the predominant association of $m c r-1$ with narrow-host types of plasmids: IncI2, IncHI2, and IncX4 (Li et al., 2017). It has been proposed the $m c r-1$ bearing IncI2 plasmids possess a fitness advantage when compared to other replicon types containing this marker (Wu R. et al., 2018). Moreover, the acquisition of IncI2-type plasmid was more beneficial for host E. coli isolate than either IncHI2 or IncX4 plasmid (Wu R. et al., 2018). Thus, the fitness advantage and co-transference/co-selection of $m c r-1$ and other antimicrobial resistance genes may further explain the fact that the most reported $m c r$-1-harboring plasmids primarily belong to the IncI2 plasmid types.

\section{Molecular Characterization and Comparative Analysis of Plasmid Backbones}

Genomic DNA (gDNA) purified from the five $m c r$-1-positive strains were subjected to sequencing using either the Illumina platform or the Pacbio RS II system. DNA of CQ9 isolated from the porcine sample in 2012 in Chongqing, was subjected to PacBio sequencing to obtain the complete genome, including,

TABLE 2 | Summary of the features associated with all 4 sequenced plasmids in CQ9 isolate.

\begin{tabular}{|c|c|c|c|c|}
\hline $\begin{array}{l}\text { Plasmid } \\
\text { number }\end{array}$ & Size (bp) & Inc type(s) & $\begin{array}{l}\text { Antibiotic resistance } \\
\text { genes }\end{array}$ & $\begin{array}{c}\text { Accession } \\
\text { number }\end{array}$ \\
\hline pCQ9-1 & 243,642 & $\begin{array}{l}\text { IncHI2 and } \\
\text { IncHI2A }\end{array}$ & sul1 and tet(A) & CP031547 \\
\hline pCQ9-2 & 221,041 & $\begin{array}{l}\text { IncFIC(FII), } \\
\text { IncFIB, IncX1, } \\
\text { and IncFII(29) }\end{array}$ & None & CP031548 \\
\hline pCQ9-3 & 189,020 & IncA/C2 & $\begin{array}{l}\text { bla } C M Y-2, \operatorname{str} A / B, \\
\operatorname{aad} A 2, \operatorname{aad} A 1, \\
\text { aph }\left(3^{\prime}\right)-l a, \operatorname{erm}(B), \\
m p h(A), \text { floR, } c m \mid A 1, \\
\text { sul2, sul3, tet(A), and } \\
\text { dfrA12 }\end{array}$ & CP031549 \\
\hline pCQ9-4 & 66,945 & Incl2 & mcr- 1 and blacTX $-M-55$ & CР031550 \\
\hline
\end{tabular}

Features include the source and size, antibiotic resistance genes, as well as plasmid Inc., types and sizes of transmissible plasmids. 


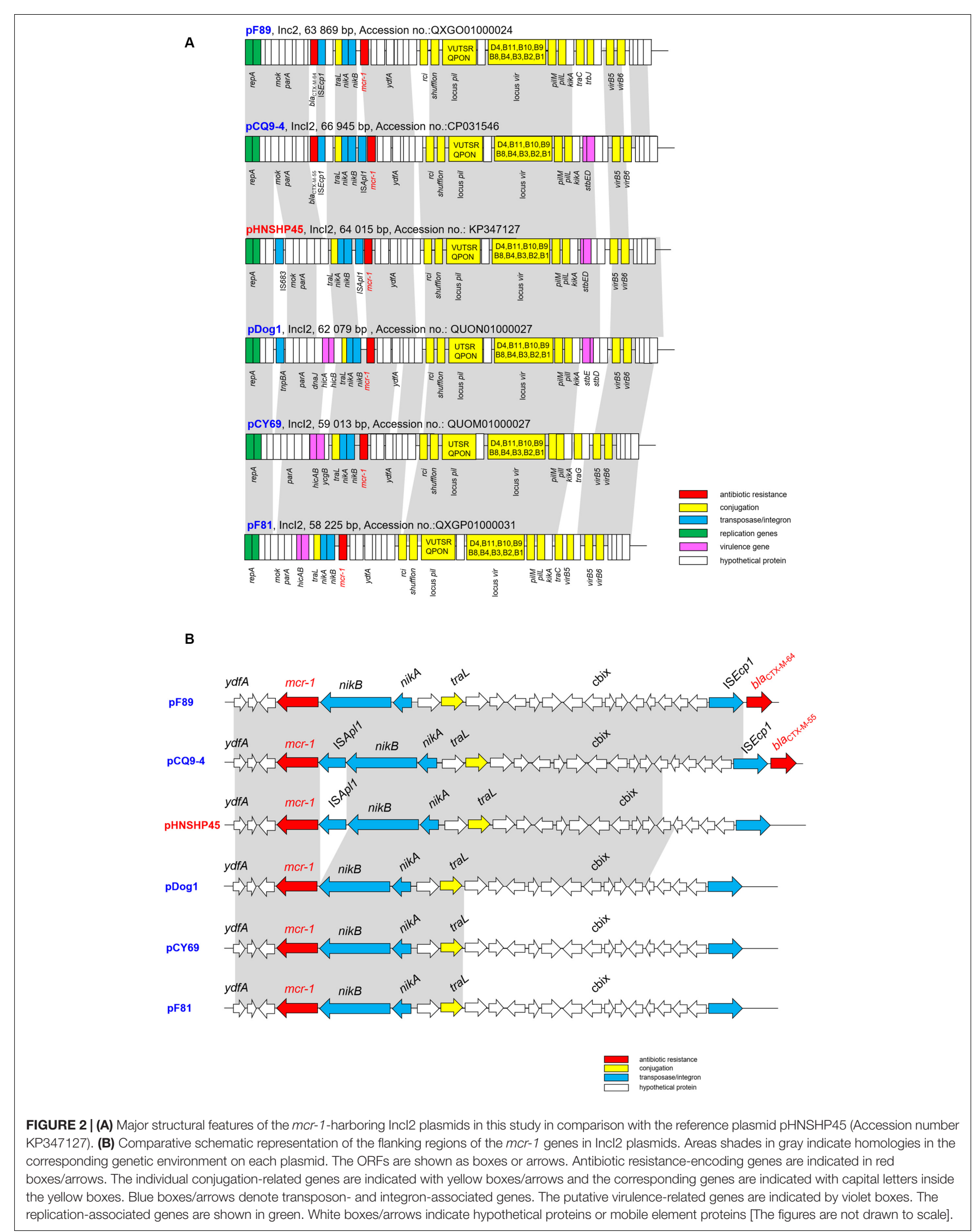


the sequences of all four plasmids (Table 2). From this isolate, the $m c r-1$ and $b l a_{C T X}-M-55$ co-harboring plasmid namely pCQ94 (CP031550), was found to be $66,947 \mathrm{bp}$ in size and exhibited a high identity (99\%) with plasmid pA31-12 isolated from a chicken isolate in Guangzhou in 2012 which highlighted the fact that these ESBL and MCR containing plasmids have been in existence and ecologically fit in different niches (Sun et al., 2016). Two of the other three complete sequences of the plasmids with large sizes derived from CQ9 had multiple resistance elements which belonged to IncHI2 and IncA/C2 (Table 2).

Complete plasmid sequences were not available for the strains sequenced using the Illumina platform, for which only the assembly scaffolds were generated. Nonetheless, the Illumina contigs of IncI2-typed conjugative plasmids recovered from F81, F89, Dog1, and CY69 isolates were obtained and compared to plasmid pHNSHP45, with results showing that these conjugative plasmids displayed high degrees of sequence homology to pHNSHP45 (Figure 2A). WGS data revealed the genetic context of the plasmids carrying $m c r-1$ in this study, had a typical plasmid backbone responsible for plasmid replication, maintenance, and transfer (Figure 2A).

When it comes to the genetic environments of the $\mathrm{mcr}$ 1 genes, none of the isolates contained Tn6330 (ISApl1-mcr1-orf-ISApl1), a structure which could generate a circular intermediate mostly found in IncHI2 $m c r-1$ plasmids (Li et al., 2017) and chromosomes (Li R. et al., 2018). Further analysis of the sequencing data obtained separately identified a 1,626-bp contig that contained the mcr-1-encoding gene. Interestingly, only one of the five plasmids (for pCQ9-4) was found to contain an ISApl1-mcr-1 cassette identical to that of plasmid pHNSHP45, whereas in the other four plasmids the mcr-1-pap cassette was inserted in a conserved position (i.e., downstream of the $n i k B$ locus) and was not associated with any flanking mobile element (e.g., ISApl1) (Figure 2B). Furthermore, a similar hypothetical protein coding sequence was detected downstream of $m c r-1$ in all plasmids, which aligned closely with phosphatidic acid phosphatase-related protein (Pap2) found in E. coli (85\% identity, GenBank accession number WP_104692553.1). The pCQ9-4 contains a complete ISApl1 insertion sequence which has its own IRL (terminal inverted repeat left) and IRR (terminal inverted repeat right), an IRR-like sequence located in pap2 (Wang Q. et al., 2016b). Although the ISApl1 cassette was not detected in another four plasmids, partial difference IRR-like sequences were still located in pap2 (Figure 2B).

The same situation has been found in Switzerland in that most of the sequenced $m c r-1$ harboring plasmids were lacking the ISApl1 element (Zurfluh et al., 2017), which is a key element mediating translocation of $m c r-1$ into various plasmid backbones (McGann et al., 2016) and chromosomes ( $\mathrm{Li} \mathrm{R}$. et al., 2018). It seems likely that complex dissemination of $m c r$-like genes relies on the diffusion of promiscuous plasmids, rather than on the clonal expansion of mcr-1-bearing bacteria. Although the prevalence of particular plasmids may vary depending on the source and geographical site, they have been increasingly isolated from bacteria of human, animal and environmental origin, highlighting the frequent exchange of genetic material between different niches.

\section{CONCLUSION}

In this study, the co-occurrence of plasmid-mediated ESBL and mcr-1 mechanisms in E. coli could be traced back to 2012 and the different MLSTs and diverse bla $a_{E S B L}$ genes in the isolates with different origins further supported that the diversified transfer mechanisms of ESBL and $m c r-1$ were present in E. coli. Moreover, our findings detected the different efficacy between cefotaxime and colistin during the conjugation experiments. When the ESBL and $m c r-1$ genes were harbored by separate plasmids in one isolate, cefotaxime could co-select both of the ESBL and $m c r-1$ genes, while the usage of colistin only selected the $m c r$-1-carrying plasmids. This observation may provide insights into one distinct manner of their dissemination; hence, further studies are needed to estimate the effects of antibioticmediated selection. Although colistin was banned for use in food animals in China, the preservation of ESBL and $\mathrm{mcr}$ 1 genes through co-selection with other antibiotics and the co-localization on a conjugative plasmid may accelerate the dissemination of both genes by HGT, which insinuated that we should pay more attention to monitoring the prevalence of plasmid mediated MDR genes.

\section{DATA AVAILABILITY}

The datasets generated for this study can be found in the Sequences were deposited in GenBank under the accession numbers: CQ9 genome (CP031546), pCQ9-1 (CP031547), pCQ9-2 (CP031548), pCQ9-3 (CP031549) and pCQ9-4 (CP031550), F81 (QXGP01000001 to QXGP01000155), F89 (QXGO01000001 to QXGO01000093), Dog1 (QUON01000001 to QUON01000107), and CY69 (QUOM01000001 to QUOM01000089).

\section{ETHICS STATEMENT}

The protocol in this study was approved by the Committee on the Ethics of Animal Care and Use of National Research Center for Veterinary Medicine (Permit 20160313088). All animal works were carried out in accordance with the recommendation of ethical guidelines of the Animal Care and Use Committee of Northwest A\&F University. Individual written informed consent for the use of samples was obtained from all the animal owners and veterinarians.

\section{AUTHOR CONTRIBUTIONS}

PZ, JW, LB, and ZY conceived and designed the study. XW, RD, $\mathrm{XB}$, and YX acquired the data. PZ, JW, JM, and LB drafted the manuscript. SF critically revised the manuscript. 


\section{FUNDING}

This work was supported in part by the National Science and Technology Infrastructure Program (2017YFC1601501), the National Natural Science Foundation of China (81402685 and 31702294), Fundamental Research Funds for the Central Universities (2452017295), Natural Science Basic Research Plan in Shaanxi Province (2018JQ3002), China Postdoctoral Science Foundation (2018T111114), China Agriculture Research System (CARS-39-14), and the Youth Innovation Team of Shaanxi Universities. The

\section{REFERENCES}

Barton, B. M., Harding, G. P., and Zuccarelli, A. J. (1995). A general method for detecting and sizing large plasmids. Anal. Biochem. 226, 235-240. doi: 10.1006/ abio. 1995.1220

Brennan, E., Martins, M., McCusker, M. P., Wang, J., Alves, B. M., Hurley, D., et al. (2016). Multidrug-resistant Escherichia coli in bovine animals. Europe. Emerg. Infect. Dis. 22, 1650-1652. doi: 10.3201/eid2209.160140

Carattoli, A. (2011). Plasmids in Gram negatives: molecular typing of resistance plasmids. Int. J. Med. Microbiol. 301, 654-658. doi: 10.1016/j.ijmm.2011.09.003

Carattoli, A., Bertini, A., Villa, L., Falbo, V., Hopkins, K. L., and Threlfall, E. J. (2005). Identification of plasmids by PCR-based replicon typing. J. Microbiol. Methods 63, 219-228. doi: 10.1016/j.mimet.2005.03.018

Clinical and Laboratory Standards Institute [CLSI], (2016). Performance Standards for Antimicrobial Susceptibility Testing; 26th Informational Supplement M100S. Wayne, PA: CLSI.

Compain, F., Frangeul, L., Drieux, L., Verdet, C., Brisse, S., Arlet, G., et al. (2014). Complete nucleotide sequence of two multidrug-resistant IncR plasmids from Klebsiella pneumoniae. Antimicrob. Agents Chemother 58, 4207-4210. doi: 10. 1128/AAC.02773-13

Dallenne, C., Da Costa, A., Decre, D., Favier, C., and Arlet, G. (2010). Development of a set of multiplex PCR assays for the detection of genes encoding important beta-lactamases in Enterobacteriaceae. J. Antimicrob. Chemother. 65, 490-495. doi: $10.1093 / \mathrm{jac} / \mathrm{dkp} 498$

Doumith, M., Godbole, G., Ashton, P., Larkin, L., Dallman, T., Day, M., et al. (2016). Detection of the plasmid-mediated mcr-1 gene conferring colistin resistance in human and food isolates of Salmonella enterica and Escherichia coli in england and wales. J. Antimicrob. Chemother. 71, 2300-2305. doi: 10. 1093/jac/dkw093

Du, H., Chen, L., Tang, Y. W., and Kreiswirth, B. N. (2016). Emergence of the mcr1 colistin resistance gene in carbapenem-resistant Enterobacteriaceae. Lancet Infect. Dis. 16, 287-288. doi: 10.1016/S1473-3099(16)00056-6

Figueiredo, R., Card, R. M., Nunez, J., Pomba, C., Mendonca, N., Anjum, M. F., et al. (2016). Detection of an mcr-1-encoding plasmid mediating colistin resistance in Salmonella enterica from retail meat in Portugal. J. Antimicrob. Chemother. 71, 2338-2340. doi: 10.1093/jac/dkw240

Haenni, M., Metayer, V., Gay, E., and Madec, J. Y. (2016). Increasing trends in $\mathrm{mcr}-1$ prevalence among extended-spectrum-beta-Lactamase-producing Escherichia coli isolates from french calves despite decreasing exposure to colistin. Antimicrob. Agents Chemother. 60, 6433-6434. doi: 10.1128/AAC. 01147-16

Kocsis, E., Guzvinec, M., Butic, I., Kresic, S., Crnek, S. S., Tambic, A., et al. (2016). blaNDM-1 carriage on IncR plasmid in Enterobacteriaceae strains. Microb. Drug Resist. 22, 123-128. doi: 10.1089/mdr.2015.0083

Li, R., Xie, M., Zhang, J., Yang, Z., Liu, L., Liu, X., et al. (2017). Genetic characterization of mcr-1-bearing plasmids to depict molecular mechanisms underlying dissemination of the colistin resistance determinant. J. Antimicrob. Chemother. 72, 393-401. doi: 10.1093/jac/dkw411

Li, R., Yu, H., Xie, M., Chen, K., Dong, N., Lin, D., et al. (2018). Genetic basis of chromosomally-encoded mcr-1 gene. Int. J. Antimicrob. Agents 51, 578-585. doi: 10.1016/j.ijantimicag.2017.11.015

Li, Y., Sun, Q. L., Shen, Y., Zhang, Y., Yang, J. W., Shu, L. B., et al. (2018). Rapid increase in prevalence of carbapenem-resistant enterobacteriaceae (CRE) and funders had no role in study design, data collection and analysis, decision to publish, or preparation of the manuscript.

\section{ACKNOWLEDGMENTS}

We thank Dr. Jingpo Cui for the kind assistance in the data analysis. The recipient strain E. coli 26R 793 was a gift from Dr. Vivi Miriagou (Laboratory of Bacteriology, Hellenic Pasteur Institute, Athens, Greece).

emergence of colistin resistance gene mcr-1 in CRE in a hospital in henan, china. J. Clin. Microbiol. 56, e1932-e1917. doi: 10.1128/JCM.01932-17

Liu, Y. Y., Wang, Y., Walsh, T. R., Yi, L. X., Zhang, R., Spencer, J., et al. (2016). Emergence of plasmid-mediated colistin resistance mechanism MCR-1 in animals and human beings in China: a microbiological and molecular biological study. Lancet Infect. Dis. 16, 161-168. doi: 10.1016/S1473-3099(15)00424-427

Lopatkin, A. J., Huang, S., Smith, R. P., Srimani, J. K., Sysoeva, T. A., Bewick, S., et al. (2016). Antibiotics as a selective driver for conjugation dynamics. Nat. Microbiol. 1:16044. doi: 10.1038/nmicrobiol.2016.44

Macrina, F. L., Kopecko, D. J., Jones, K. R., Ayers, D. J., and McCowen, S. M. (1978). A multiple plasmid-containing Escherichia coli strain: convenient source of size reference plasmid molecules. Plasmid 1, 417-420. doi: 10.1016/0147-619x(78) 90056-2

McGann, P., Snesrud, E., Maybank, R., Corey, B., Ong, A. C., Clifford, R., et al. (2016). Escherichia coli harboring mcr-1 and blaCTX-M on a novel IncF plasmid: first report of mcr-1 in the United States. Antimicrob. Agents Chemother. 60, 4420-4421. doi: 10.1128/AAC.01103-1116

Michalopoulos, A. S., Tsiodras, S., Rellos, K., Mentzelopoulos, S., and Falagas, M. E. (2005). Colistin treatment in patients with ICU-acquired infections caused by multiresistant Gram-negative bacteria: the renaissance of an old antibiotic. Clin. Microbiol. Infect. 11, 115-121. doi: 10.1111/j.1469-0691.2004.01043.x

Paterson, D. L., and Harris, P. N. (2016). Colistin resistance: a major breach in our last line of defence. Lancet Infect. Dis. 16, 132-133. doi: 10.1016/S1473-3099(15) 00463-466

Poirel, L., Kieffer, N., Brink, A., Coetze, J., Jayol, A., and Nordmann, P. (2016). Genetic features of MCR-1-producing colistin-resistant Escherichia coli isolates in South Africa. Antimicrob. Agents Chemother. 60, 4394-4397. doi: 10.1128/ AAC.00444-416

Rozwandowicz, M., Brouwer, M. S. M., Fischer, J., Wagenaar, J. A., Gonzalez-Zorn, B., Guerra, B., et al. (2018). Plasmids carrying antimicrobial resistance genes in Enterobacteriaceae. J. Antimicrob. Chemother. 73, 1121-1137. doi: 10.1093/jac/ $\mathrm{dkx} 488$

Schwarz, S., and Johnson, A. P. (2016). Transferable resistance to colistin: a new but old threat. J. Antimicrob. Chemother. 71, 2066-2070. doi: 10.1093/jac/dkw274

Shen, Y., Zhou, H., Xu, J., Wang, Y., Zhang, Q., Walsh, T. R., et al. (2018). Anthropogenic and environmental factors associated with high incidence of mcr-1 carriage in humans across China. Nat. Microbiol. 3, 1054-1062. doi: 10.1038/s41564-018-0205-208

Shen, Z., Wang, Y., Shen, Y., Shen, J., and Wu, C. (2016). Early emergence of mcr1 in Escherichia coli from food-producing animals. Lancet Infect. Dis. 16:293. doi: 10.1016/S1473-3099(16)00061-X

Sun, J., Li, X. P., Yang, R. S., Fang, L. X., Huo, W., Li, S. M., et al. (2016). Complete nucleotide sequence of an IncI2 plasmid coharboring blaCTX-M-55 and mcr-1. Antimicrob. Agents Chemother. 60, 5014-5017. doi: 10.1128/AAC.00774-716

Sun, J., Zhang, H., Liu, Y. H., and Feng, Y. (2018). Towards understanding MCRlike colistin resistance. Trends Microbiol. 26, 794-808. doi: 10.1016/j.tim.2018. 02.006

Sun, P., Bi, Z., Nilsson, M., Zheng, B., Berglund, B., Stalsby, L. C., et al. (2017). Occurrence of blaKPC-2, blaCTX-M, and mcr-1 in Enterobacteriaceae from well water in rural China. Antimicrob. Agents Chemother. 61, e2569-e2516. doi: 10.1128/AAC.02569-2516

Wang, J., Hurley, D., McGrath, K., Bai, L., Hachler, H., Stephan, R., et al. (2016a). Draft genome sequence of Escherichia coli 26R 793, a plasmid-free recipient 
strain commonly used in conjugation assays. Genome Announc. 4, e707-e716. doi: 10.1128/genomeA.00707-716

Wang, Q., Li, Z., Lin, J., Wang, X., Deng, X., and Feng, Y. (2016b). Complex dissemination of the diversified mcr-1-harbouring plasmids in Escherichia coli of different sequence types. Oncotarget 7, 82112-82122. doi: 10.18632/ oncotarget.12621

Wang, J., Stephan, R., Karczmarczyk, M., Yan, Q., Hachler, H., and Fanning, S. (2013). Molecular characterization of bla ESBL-harboring conjugative plasmids identified in multi-drug resistant Escherichia coli isolated from food-producing animals and healthy humans. Front. Microbiol. 4:188. doi: 10.3389/fmicb.2013. 00188

Wu, C., Wang, Y., Shi, X., Wang, S., Ren, H., Shen, Z., et al. (2018). Rapid rise of the ESBL and mcr-1 genes in Escherichia coli of chicken origin in China, 2008-2014. Emerg. Microbes Infect. 7:30. doi: 10.1038/s41426-018-0033-31

Wu, R., Yi, L. X., Yu, L. F., Wang, J., Liu, Y., Chen, X., et al. (2018). Fitness advantage of mcr-1-bearing IncI2 and IncX4 plasmids in vitro. Front. Microbiol. 9:331. doi: 10.3389/fmicb.2018.00331

Zurfluh, K., Nüesch-Inderbinen, M., Klumpp, J., Poirel, L., Nordmann, P., and Stephan, R. (2017). Key features of mcr-1-bearing plasmids from Escherichia coli isolated from humans and food. Antimicrob. Resist. Infect. Control 6:91. doi: 10.1186/s13756-017-0250-258

Zurfluh, K., Tasara, T., Poirel, L., Nordmann, P., and Stephan, R. (2016). Draft genome sequence of Escherichia coli S51, a chicken isolate harboring a chromosomally encoded mcr-1 gene. Genome Announc. 4, e796-e716. doi: 10.1128/genomeA.0079 6-716

Conflict of Interest Statement: The authors declare that the research was conducted in the absence of any commercial or financial relationships that could be construed as a potential conflict of interest.

Copyright (c) 2019 Zhang, Wang, Wang, Bai, Ma, Dang, Xiong, Fanning, Bai and Yang. This is an open-access article distributed under the terms of the Creative Commons Attribution License (CC BY). The use, distribution or reproduction in other forums is permitted, provided the original author(s) and the copyright owner(s) are credited and that the original publication in this journal is cited, in accordance with accepted academic practice. No use, distribution or reproduction is permitted which does not comply with these terms. 